\title{
A Qualitative Investigation into Practitioners' Perspectives of the Coping-Infused Dialogue through Patient-Preferred Live Music Protocol
}

\author{
Michael Joseph Silverman ${ }^{1 *}$ \\ 1 University of Minnesota, United States \\ *silvermj@umn.edu \\ Received: 5 April 2017 ; Accepted: 7 September 2017 ; Published: 1 March 2018 \\ Editor: Helen Oosthuizen. Reviewers: Alison Talmage, Charlotte Lindvang
}

\begin{abstract}
The Coping-Infused Dialogue through Patient Preferred Live Music (CID-PPLM) protocol was designed to integrate receptive music therapy with a discussion of stressors and coping skills. However, to better understand the advantages and disadvantages of the protocol within the contemporary evidence-based practice framework, investigation with protocol practitioners is warranted. The purpose of this study was to understand practitioners' perspectives of the CID-PPLM protocol. Specific research questions included practitioners' perspectives of the following: (1) What are potential advantages of the CID-PPLM protocol and how might it function with adult medical patients? (2) What are potential disadvantages of the CID-PPLM protocol and how might it be improved? Five practitioners participated in individual semi-structured interviews. Themes were identified via thematic analysis. Member checking and trustworthiness were used. Participants had positive, as well as constructive, perceptions of the CID-PPLM. Emerging themes included: CID-PPLM provides choice, control, support, and autonomy; CID-PPLM allows for individualized patient responses within a distinct therapeutic interaction; and the CID-PPLM can be restrictive. Emerging themes and sub-themes can be used to modify the CID-PPLM and provide a framework for new protocols to offer clinicians additional flexibility to best serve adult medical patients. Implications, limitations, and suggestions for future research are provided.
\end{abstract}

Keywords: coping, CID-PPLM, interview, patient preferred live music, music therapy, qualitative

\section{Literature Review}

As hospitalized patients on medical units may feel fatigued or unwell, they typically desire a receptive music therapy experience as opposed to an intervention wherein they are actively required to be engaged, on task, and participating. Additionally, patients may not have the physical strength or motivation to participate in an active music making or creating experience. Patient-preferred live music (PPLM) is a receptive music therapy intervention and often constitutes an ideal and preferred intervention for adult hospitalized medical patients. As such, PPLM can be operationally defined as a receptive music therapy experience involving music selected and preferred by the pa- 
tient and performed live by a qualified music therapist. In a recent systematic review concerning PPLM with adult medical patients, quantitative studies that met the inclusion criteria were encouraging and supported PPLM (Silverman, Letwin, \& Nuehring, 2016). The authors noted that PPLM, when delivered by trained music therapists, can be an effective intervention for affective states, pain, nausea, and physiological measures.

Although PPLM may have immediate positive affective results, adult medical patients have long-term and complex needs adapting to life after major medical procedures. One approach clinicians use to help patients manage psychosocial stressors in the medical setting is teaching patients to identify stressors and use effective coping strategies. Coping can be defined as cognitive and behavioral efforts to manage stressful events (Lazarus \& Folkman, 1984) and represents an important component of stress research (Lazarus, 2006; Semmer \& Meier, 2009) because individuals who use effective coping can maintain or improve perceived wellbeing during stressful events (Lazarus \& Folkman, 1984). While coping skills can be a central factor in mitigating the impact of stressful events, patients may have inadequate knowledge of coping skills to proactively and reactively manage stress. By increasing patients' knowledge of and ability to use effective coping skills, medical professionals may improve outcomes by reducing psychosocial stressors and resultant negative affective states and outcomes in their patients.

As physicians or nurses often have little time to talk with medical patients about psychosocial stressors they confront in and outside of the hospital, researchers developed the Coping-Infused Dialogue through Patient Preferred Live Music (CID-PPLM) protocol for the purpose of integrating the discussion of stressors and coping skills within a single receptive music therapy session in an inpatient medical setting (Hogan \& Silverman, 2015). Designed by Board-Certified Music Therapists, this protocol was intended for practitioners to be able to offer a structured interaction concerning potential stressors and coping skills when working with adult medical patients. Based on the integration of Robb's Contextual Support Model of music therapy (2003) and positive results of PPLM (Silverman, Letwin, \& Nuehring, 2016), the CID-PPLM was designed to provide three forms of contextual support through music: 1) structure via familiar music and a predictable session structure alternating between live music and dialogue; 2) autonomy support via patient selection of preferred music and topics for dialogue; and 3) relationship support via shared music interactive experiences. These forms of contextual support were intended to augment patient engagement and foster dialogue concerning local and global stressors and coping skills. The purpose of the original randomized controlled CID-PPLM pilot study was to measure the effects of the protocol on positive and negative affect and pain in hospitalized solid organ transplant patients (Hogan \& Silverman, 2015). Results $(N=25)$ indicated significant between-group differences in positive affect, negative affect, and pain, with experimental participants having more favorable posttest scores than control participants. As many patients often are only hospitalized for a few days in the United States and thus only receive minimal exposure to music therapy (Boyle, 2008; Miller, 2008), it should be stressed that the intent of the single-session CID protocol is not necessarily enhanced coping from a behavioral perspective but helping patients to cognitively identify their problems and potential solutions to these problems in both local (i.e., within the hospital) and global (i.e., outside the hospital) contexts. A rationale for and description of the CID-PPLM intervention protocol can be found in Hogan and Silverman (2015). Additionally, a related follow-up study comparing PPLM only and CID-PPLM found that the addition of CID did not adversely impact affective state or pain (Silverman, Nuehring, \& Letwin, 2016). As initial results of quanitative studies support the CID-PPLM protocol, additional investigations using various paradigms and data types are warranted.

Evidence-based practice (EBP) often guides practitioners towards making informed clinical decisions that are patient-centered and research based. EBP is the conscientious integration of (a) the best research available, (b) the clinician's expertise, and (c) the patient's characteristics, culture, values, and preferences (Rubin, 2008; Sackett, 
Straus, Richardson, Rosenberg, \& Haynes, 2000). As music therapy clinicians can be considered experts in their profession, their opinions constitute a component of EBP. Thus, practitioners' perspectives of their interventions can complement the best research evidence available and the patient's characteristics, culture, values, and preferences in order to make care-related decisions. However, the Centre for Evidence Based Medicine (2017) noted that the practitioner's opinions constitute the lowest type of evidence within the levels of evidence hierarchy. Although the other two components of EBP are typically emphasized, it is also important to understand practitioners' perceptions of their interventions, as they possess clinical expertise (Centre for Evidence Based Medicine, 2017). To date, there is a lack of scientific exploration concerning practitioners' perspectives of formalized and published music therapy protocols, such as the CID-PPLM. Within the EBP framework, practitioners' perspectives are valuable and such data could improve current protocols and identify factors to be included in new protocols.

To better understand the advantages and disadvantages of the CID-PPLM protocol from the practitioners' perspectives within the EBP framework, it would seem appropriate to conduct an investigation with the music therapists who have used the protocol. Therefore, the purpose of this study was to understand practitioners' perspectives of the CID-PPLM protocol. Specific research questions included practitioners' perspectives of the following:

1. What are potential advantages of the CID-PPLM protocol and how might it function with adult medical patients?

2. What are potential disadvantages of the CID-PPLM protocol and how might it be improved?

\section{Method}

\section{Participants}

Research participants were five Board-Certified Music Therapists or second year music therapy graduate students. All participants had delivered the CID-PPLM protocol to adult medical inpatients who were hospitalized on solid organ transplant or cancer units. All participants had been supervised by the author. Thus, purposive sampling was used and the researcher acknowledges his prior relationship with participants. All participants volunteered to take part in the study and, after the researcher explained the study, signed informed consent forms. Participants did not receive payment for their participation.

\section{Author Lens and Biases}

At the onset of data collection, the researcher had over 15 years of music therapy clinical experience and had worked with participants from two to four years. The researcher emphasized the importance and purpose of the study was to further understand the advantages and disadvantages of the protocol and therefore encouraged participants to be as honest as possible during interviews. As participants had a previously established professional relationship with the researcher, it was hoped that they were able to speak openly and truthfully. As evidenced by the participants' critical comments during interviews, the researcher was confident that participants felt enabled to critique and challenge the protocol.

The researcher was a Board-Certified music therapist practicing in the United States whose primary philosophical orientation is cognitive behavioral (Craske, 2010; Mansell, Carey, \& Tai, 2013). The researcher has published refereed journal articles relating to medical music therapy (Fredenburg \& Silverman, 2014; Haack \& Silverman, 2016; Letwin \& Silverman, 2017; Rosenow \& Silverman, 2014; Yates \& Silverman, 2015). During the interviews, the researcher actively involved participants by asking them to elaborate on statements and engaging them in detailed conversations in an 
attempt to deeply understand their perceptions and experiences. The gestalt of these factors informed the researcher's thought processes, ways of knowing, and interpretation of the data (Edwards, 2012; Stige, Malterud, \& Midtgarden, 2009).

\section{Procedure}

The researcher used semi-structured interviews (see Appendix) consisting of pre-determined questions that functioned as guides and prompts during the interview process. The interviews were purposely broad in scope due to the lack of existing literature concerning the CID-PPLM. Throughout the interviews, the researcher spontaneously asked probe questions to further and deeply understand participants' perceptions and experiences. In an attempt to encourage participants to speak freely and for the discussion to be as organic as possible, the researcher was flexible with the organization of the questions during the semi-structured interviews. Individual interviews lasted from approximately 30 to 60 minutes. Interviews were video and audio recorded and transcribed by a research assistant. During the interviews, the researcher took reflexive notes concerning both participants' statements and his reactions to these statements. The purpose of these notes was to develop follow-up questions for additional depth, but these notes were not included in data analysis. This project was approved by the author's affiliated institution (University of Minnesota Institutional Board Code Number: 1504S68621).

\section{Qualitative Analysis}

The researcher used an inductive approach to thematic analysis to identify themes from transcribed data, wherein initial codes were directly linked to the data but were not driven by the researcher's a priori assumptions concerning relationships among or within data. The author identified and established code categories and themes during repeated readings of the data. Coding began after all interviews were completed due to the low number of participants and the desire to avoid any preconceptions. The process of data analysis was based upon Braun and Clarke's (2006)'s six phases of thematic analysis for researchers, comprising a) familiarization with the data; b) generation of initial codes; c) searching for themes; d) reviewing themes; e) defining and naming themes; and f) producing the report.

During the analysis, the researcher coded participants' quotes in margins of the transcript. The researcher then copied and pasted quotes and codes into a separate document and organized quotes by their codes. Then, the researcher grouped similar codes from different participants together and, after repeated readings and edits of the codes, themes emerged from these related code categories. This process allowed the researcher to compare and contrast related codes among participants.

\section{Member checking and trustworthiness}

After transcription of the interviews, the researcher read transcriptions, designed follow-up questions where appropriate, and sent the transcriptions and follow-up questions to participants. Participants were asked to read, make clarifications, comment on, and answer follow-up questions from the transcribed interviews. After thematic analysis, the researcher emailed the resultant themes, subthemes, and quotes used to depict themes to participants. Participants were asked for feedback and provided constructive as well as supportive comments. After receiving and integrating these comments into the manuscript, the researcher described and presented the project to nine students in a graduate level medical music therapy class. These students provided critical and supportive feedback and verified themes and the codes used to support the themes. Feedback from the graduate level medical music therapy class was integrated into the results. Finally, the researcher requested feedback from a peer researcher (not affiliated with the current study) who has published numerous articles in peer-reviewed jour- 

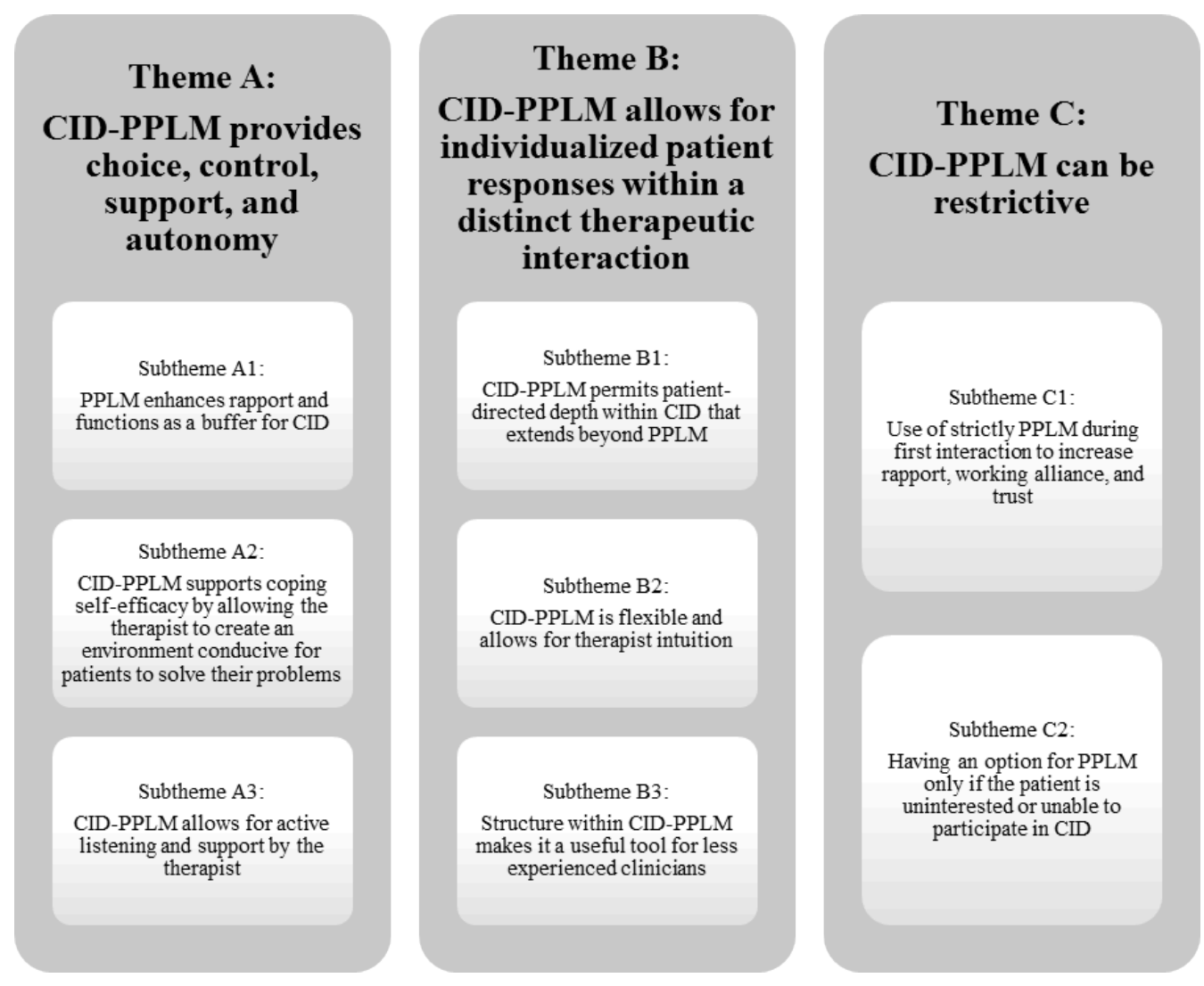

Figure 1

Depiction of three themes and eight subthemes

nals. The peer researcher also provided constructive and supportive feedback which was integrated into the results.

\section{Results}

Themes A and B (and six supporting subthemes) answered research question 1 and Theme C (and two supporting subthemes) answered research question 2. Themes and sub-themes are depicted in Figure 1 to provide a holistic depiction of the results. Identified codes and anonymized participant quotes are included with each subtheme to provide transparency to data analyses and honor the experiences and perspectives of the participants, who had expertise in delivering the CID-PPLM protocol.

\section{Research Question 1: What are potential advantages of the CID-PPLM protocol and how might it function with adult medical patients?}

Themes A and B represent participants' perspectives of the advantages of the CIDPPLM. Themes are described in detail below and, in an attempt to honor the participants' unique voices and clinical expertise congruent within the EBP framework, participants' quotes are depicted to support the subthemes.

\section{Theme A: CID-PPLM provides choice, control, support, and autonomy}

In describing advantages of the CID-PPLM, participants tended to separate the PPLM and CID components in their interviews. They noted a sequential process wherein the receptive and choice-driven aspects of the PPLM first functioned to immediately enhance rapport that then facilitated the transition to the more therapeutic aspect of the CID. The encouraging environment facilitated by the PPLM provided an opportunity for the practitioners to support patients' coping self-efficacy and allowed the patients a safe space to identify potential ways to deal with their stressors. Additionally, as no 
other hospital staff used music with patients, the protocol using PPLM was unique and facilitated development of the therapeutic relationship between the practitioner and the patient. Thus, the protocol allowed music therapists to have a unique music-based interaction with patients that differed from other hospital staff. During the exploration of potential coping skills, the protocol allowed practitioners to be active and engaged listeners and support patients as they explored potential coping strategies rather than provide practitioner-driven strategies. Participants recognized the busy schedules of other hospital staff and how the patients desired additional interactions that the music therapists could fulfill.

\section{Subtheme A1: PPLM enhances rapport and functions as a buffer for CID.}

Codes: rapport, buffer, relationship, music-based interaction

- ...the idea was, basically, that the patient-preferred live performance element of the protocol was something that would help both rapport and help the patient talk to the therapist more and just kind of provide an "in," so that you could get a conversation going... we know that music can be a very great way to initiate, develop, and facilitate social interaction in that if you see someone playing music and giving that experience to you, it can just open up a level of rapport that's either just deeper or developed more quickly than you would otherwise be able to do in a hospital room. (A)

-...PPLM is the easiest way to build rapport with someone and prove that you understand them and you're not there to play music that you enjoy but that you actually play what they enjoy...I think having that buffer with the CID makes it...if I went in and just asked them point blank what their stressors were, I don't think it would maybe come off as well...but I think having the CID sort of paired with the PPLM makes it easier to get it out... (M)

- I really think the rapport is a really important part of this...it is in the beginning of the protocol to discuss music preferences...I felt that the music helped the therapeutic relationship grow and become comfortable in most situations. Playing a couple songs at the beginning of the session may "break the ice" and help patients open up...(R)

\section{Subtheme A2: CID-PPLM supports coping self-efficacy by allowing the therapist to create an environment conducive for patients to solve their problems.}

Codes: support, self-efficacy, facilitate

- ...ultimately it's the therapist's job to help the patient find his/her own coping strategy, because I can't just come in with a list of coping strategies and tell the patient, "Okay, you need to choose this strategy or use that strategy," because that's not going to be functional for the patient. The patient needs to find a strategy that's going to work for their own life. (A)

- ...but really my goal was just to help them identify some way of coping or identify ways that they already do it, anything like that...but I was always very cautious about coming in and not trying to fix anything but more just opening up the door for them to talk about it and allowing them, more than just suggesting new coping skills for them to use, more like let them talk about it and see, "Oh, this is something that I'm already doing that I do find as a good way to cope," and identifying it in that way. $(\mathrm{H})$

- So I think it works in the fact that it helps people simply think about it. And it's a nice way to get people's minds maybe realizing that "Oh, this is something that I could actually do something about" or "There is ways that I can take care of this 
at home." So I think of, when people have the opportunity to see that it is changeable and that they're not necessarily stuck with it, helps their minds figure out, "well, what are some options?"...So having, help people get that belief, I think, is simple in the fact that we're not making them do anything but help them believe that they are capable of doing something...it's not intended to be a deep "pull out all of your secrets" isn't intended to happen within, you know, 20 to 30 minutes and then they have one or two things that they can consider, not ensured that they're going to do, but something that they can consider implementing. (M)

- And, in doing the dialogue with patients, for the most part, I wasn't giving them new information, but focusing on having them identify what they already know and then reinforcing the coping strategies that they were identifying...(U)

\section{Subtheme A3: CID-PPLM allows for active listening and support by the therapist}

Codes: listen, support, talk, share

- ...they really need someone to talk about these stressors with because the nurses on the floor are wonderful, but they don't have just one patient, they have, you know, maybe four or five. So, having the time to sit down with them and chaplains can't come every day because there's maybe two chaplains or what not. (M)

- I also thought it was a great outlet for the patients to just express themselves because they really just want someone there to listen to them and talk with them....at the end of the session some patients would tell me, "You know, I really appreciate you coming in here and talking to me and listening to me because this has been a very rocky experience." Like someone going through their hair being shaved, that was really kind of terrifying for them and they weren't sure who to talk to about it and so I would be in there and they would just kind of come and express to me what their stressors and by the end they would always say "I feel much more relieved and much more relaxed than I was at the beginning of the session." So, some did express that, the dialogue did help to calm their nerves and some just said "Wow, that was really great. I just wanted someone to talk to." (R)

- But then I also think a lot of them, especially those who were maybe farther from home or who don't have as many visitors, just really like having someone come in and talk to them, who's not going to ask them to do stuff or be there to give them a shot or that kind of thing, but just to have someone who's there to chat about their experience and actively listen. And I think that was helpful for a good portion of them as well...and I think the support was beneficial as well. As I said earlier, to have someone just come in who is just there to talk and play some music and actively listen. You know, maybe they don't have a lot of visitors coming in, so it gives them an opportunity to talk about their experience. (U)

\section{Theme B: CID-PPLM allows for individualized patient responses within a distinct therapeutic interaction}

Participants had positive perceptions of the way the CID-PPLM protocol allowed patient-directed responses within a unique interaction. They noted the protocol allowed the patient to direct the level of sharing and depth of the dialogue. Moreover, due to the identification of stressors and ways to deal with stressors, this dialogue was more personal - and could be potentially more therapeutic - than the dialogue within a PPLM-only intervention. Participants noted that despite being a protocol, the CIDPPLM contained elements of flexibility wherein the practitioners could rely on their intuition as music therapists. At the same time, participants noted that the protocol may be ideal for less experienced music therapists, or student music therapists who are developing therapeutic interaction skills, due to the structure within the CID-PPLM. 


\section{Subtheme B1: CID-PPLM permits patient-directed depth within CID that extends beyond PPLM}

Codes: depth, patient decision, further, advance

- ...with that rapport that you can develop in a matter of minutes rather than a matter of days, the theory or idea behind the protocol is that you're able to dig deeper into conversation within a half-hour to forty-five minute time span that you wouldn't otherwise be able to. So, the idea of short, efficient treatment is one of the hallmarks of the CID-PPLM. A music therapist comes in, establishes rapport quickly through a prescribed patient-preferred music model, and then is able [to] talk about the challenges and life issues that maybe no one else in the treatment team can get within a half hour time span. And then from that point, the copinginfused dialogue is really meant to, be the open conversations where patients feel safe and they feel welcome to talk about things that are difficult for them, why they're in the hospital, and then things that they might be struggling with outside of the hospital and just helping the patient identify what coping strategies may be effective for some of those problems... (A)

- I think that prepares the patient, starting where they're at and starting small. And then, if they're willing to go with you, you go into maybe some deeper issues and deeper stressors...(H)

-...it's nice to have an opportunity to do something that's more than just one of your favorite songs/what concerts have you gone to. It's nice to have a pool to pull from that lets you go a little deeper...it's a great way to think about "what are your stressors - locally, globally" but incorporate in more harmoniously/congruently within the session so it doesn't feel so abrupt...because even when I was in my internship, I still felt the things at the hospital were still pretty PPLM-based; but to add an extra layer or even just add an opportunity to talk about stressors and things that they can possibly deal with is just a nice thing to add on when you have the right client and have the rapport established already. (M)

- And another thing that I liked about it is that the patients can direct how in-depth they want it to go...And so they can direct what level they want to take the dialogue to and then I have room within the protocol to respond to where they're going...one of the things that they probably like about the protocol is that it is noninvasive....and they can choose where they want that conversation to go...Again, patients choose how deep they want the dialogue to go. (U)

\section{Subtheme B2: CID-PPLM is flexible and allows for therapist intuition}

Codes: flexible, structure, intuition, idiosyncratic, change, adjust

- But at the same time, it's somewhat a flexible or living document which provides you a helpful guideline without necessarily locking you into a predetermined script of what you have to say to the patient...that's one of the strengths and weaknesses of the protocol, is that the flexibility gives you the opportunity to adjust the intervention based on how your patient responds...I probably adjusted the amount of talk about coping skills and/or verbal processing based on the mood of the patient or body language I was getting, just with the understanding that everyone is kind of in a different place and they have a different level of willingness to participate in this kind of activity. (A)

- ...what I like about the mechanisms is that it doesn't say how much talking, I mean, it says to alternate talking and the song, but it doesn't really say how long the discussions have to be. So, if there needs to be more music, the little conversations in between can be shorter, and if it needs to be more conversation, uh, the dialogue parts can be longer...there's a little bit of flexibility built into it. $(\mathrm{H})$

- And also, if they don't feel comfortable sharing with me and I force them to share with me then that's not an appropriate way to establish therapeutic rapport. And I 
think that's true with really any approach you take, that you're not gonna go into a setting and just use a cookie cutter approach...you have to see what they have and what they present with... (M)

- ...so just having those main tasks and goals within the protocol was good but I could also kind of elaborate upon it or even if the local stressors came first or the global came first, whatever the patient is ready to talk about... it has a nice outline that can also be diverse within itself...I think it provides a really great guideline, which can also be really flexible at the same time...So, I sometimes kind of framed the wording a little bit differently depending on the patient... (R)

- ... if they're just not opening up and I've given them a couple prompts and they're sort of indicating to me that they're not feeling confident, I can go back to the music. That doesn't mean that the protocol is ineffective. I liked having the structure and having a guideline for where I want to go with the conversation...Yes, I like that it has structure but also flexibility and it's not rigid and it's not, you know, it's not a script...So, it's not "ask this question and then ask this question and then follow up with this"...I was able to adapt it in each session. (U)

\section{Subtheme B3: Structure within CID-PPLM makes it a useful tool for less experienced clinicians}

Codes: safe, experience, simple, structure, flow, guide, inexperienced

- ...in terms of protocol, one of the things that I really like about it is that it provides a very clear guideline for clinicians. It has a goal in mind that I think most people who read the protocol can instantly understand - "Oh yeah, this is where the therapy session should be going and this is where the dialogue that I'm choosing to use should be going"...And, while some of these potential scripts or statements about what the goal of the conversation should be... while that may seem very intuitive or obvious to experienced clinicians or to clinicians who, understand therapy very clearly, this guideline, I think, can be very useful for younger clinicians who maybe go into a hospital room and don't know what to say to someone. (A)

- The thing I like about the protocol is that the clinician or researcher always kind of has a next step in mind...I think it gives the researcher or clinician an idea; they always have the next step in mind. So, it kind of acts as a safety net for you. I like the idea of starting with the local and going to the global stressors and coping...I consider myself an inexperienced clinician and from personal experience I think it's good and I'd use that idea...I mean, I definitely use that idea of starting shallow and going deep with whatever topic you're on. But yeah, you're talking about specifically this protocol, and I do think any handbook approach is going to make it easier, especially for real beginners. $(\mathrm{H})$

- ...it's simple in the fact that it gives you two things to talk about. So, if you don't feel that you have a lot of, I think, a bag of tricks of helping with therapy or how to talk to people and some of those talk-therapy type things. I think it's a good way to ask questions that could dig deeper if you follow up with the right questions...I think it's a good way to have some springboard and something for music therapists with less experience to have two questions that they could at least ask and have some options to follow up with...I do believe this is a nice tool for newer/less experienced music therapists who don't have the counseling skills yet. Experienced music therapists can definitely use it, but I think it's a nice use for newer music therapists want to go deeper than PPLM, but haven't had years of experience yet with talking to people. It would have been nice to use during my internship for sure not only in the hospital but hospice as well. It's a nice gateway to deeper counseling skills and discussions, but it can also be just about stressors and thoughts/actions too. (M) 
- I think for those that maybe are in school and not as experienced as a clinician in the field already, possibly in the medical field specifically...So, I think it's definitely adaptable for those that aren't as experienced, just because it is laid out so nicely and it's also simple to understand what the target is and what the end result should be. (R)

-...it was really helpful for me as an inexperienced clinician to have the structure and the prompts that it provided...Again, certainly the structure for someone who doesn't have as much clinical experience. (U)

\section{Research Question 2: What are potential disadvantages of the CID- PPLM protocol and how might it be improved?}

Theme $C$ represents participants' perspectives of the disadvantages and methods for improving the CID-PPLM protocol.

\section{Theme C: The CID-PPLM can be restrictive}

Participants articulated ways in which the CID-PPLM may be improved. Similar to Theme A, practitioners tended to separate the CID from the PPLM when articulating these concepts. First, practitioners noted that the protocol might be extended to a 2-day intervention wherein day one only contained PPLM to specifically develop rapport, alliance, and trust. By purposely excluding CID on the first day of a 2-day intervention, practitioners theorized that patients would be more apt to share personal stressors and coping strategies during the second day of the protocol, wherein the entire CID-PPLM could be implemented. Practitioners also suggested that the protocol be modified to include a path to avoid CID altogether and solely use PPLM should the patients not respond well to the CID.

\section{Subtheme C1: Use of strictly PPLM during first interaction to increase rapport, working alliance, and trust}

Codes: invasive, quick, intimidate, slow down, rush

- It's great for a second session but it also feels a bit invasive for a first session...I don't think the PPLM itself would be invasive if it was a second session or a third session but a lot of these people, when I would go in and introduce myself, I'd have to say my affiliations, say I was a graduate student...I also look really young so I don't know how much they trust me and there's no therapeutic rapport established whatsoever...but I still think it seems really sort of invasive for a first session as a meeting with someone and figure out what their music is but also, kind of pull out of them what's stressing them...so if we had done it a second session or even a third, it would have had a greater opportunity to work and see what would happen. (M)

- So, maybe having a day dedicated to that or maybe a longer time period dedicated to that, maybe discussing some areas that we could start plugging in how to build rapport at the beginning of the session, even more than what was already done because some patients were super ready to just jump in and talk where others I could tell they weren't as comfortable and then I would go back and visit them on day two or day three just on my own time and it would be a little bit different compared to the first time I saw them... (R)

- I have half an hour to forty-five minutes to build that relationship, use the music, and open up their dialogue. And it was enough time, but there were times when I felt like more time would have been helpful, you know. If I had had either a little longer with each patient or an additional session so that I could focus on building rapport with the music before getting into the dialogue... (U) 


\section{Subtheme C2: Having an option for PPLM only if the patient is uninterested or unable to participate in CID}

Codes: PPLM only, no CID, quiet, non-responsive

- Yeah, that would definitely be an option and a very, sort of, multi-linear path that clinicians could follow if they didn't quite know how to keep the dialogue moving just based on the existing protocol. (A)

- There were times when I think the patients really just wanted to just hear music and not even dialogue at all between songs and you can feel that pretty quick. You know, after the first song and you start conversing with them a little bit, it would seem like they were just ready to get back to the music... when they didn't want the discussion component. $(\mathrm{H})$

-... those trouble spots that I ran into (patients not wanting to talk, difficulty transferring music/lyrics to the discussion, etc.) needed more preparation or back up options to work from...just when a patient isn't ready to discuss, their life with you or their experiences with you...but if that ever does happen within the protocol, what's the next step that could be done in case patients are ready for that should I just go straight to PPLM, should I continue to talk and include the talking? ... But, yeah just something to kind of have as a backup if that does happen - where's the next step in the flow chart to go if it's not working? (R)

-...maybe they were taking too many pain medications, or whatever, to be able to participate in the dialogue... (U)

\section{Discussion}

The purpose of this study was to understand practitioners' perspectives and experiences with the CID-PPLM protocol in order to determine advantages and disadvantages of the protocol. Three themes (CID-PPLM provides choice, control, support, and autonomy; CID-PPLM allows for individualized patient responses within a distinct therapeutic interaction; and the CID-PPLM can be restrictive) and eight supporting sub-themes emerged. Generally, practitioners had both positive and constructive perceptions of the CID-PPLM.

Subtheme C2 (having an option for PPLM only if the patient is uninterested or unable to participate in CID) is particularly relevant from a clinical perspective. As all practitioners were involved in delivering the CID-PPLM via various research projects, this factor highlights the challenging discrepancy between clinical work and controlled research. During clinical work, practitioners have considerably more freedom to change the intervention based upon client response and need. Thus, if a patient was not responsive to the CID component within the protocol, the clinical practitioner could alter the session to include more PPLM. However, from a controlled empirical perspective, research practitioners did not have that flexibility to change the intervention from CID-PPLM to PPLM only. This subtheme underscores that participants - who had all previously conducted quantitative studies using the CID-PPLM protocol - identified complications resulting from using protocols and tightly controlled independent variables in clinically-based effectiveness research.

One of the incongruences of the results concerned subtheme B3 and theme C. Participants noted that the protocol contained structure that functioned as a type of safety net for practitioners who had less clinical experience (subtheme B3) but also felt hindered by lacking additional flexibility that was needed if the patient was unreceptive to the CID component of the CID-PPLM (theme C). Additionally, participants noted a need for additional time to develop rapport with some patients by using PPLM only. In these cases, only using PPLM was the preferred option. There was also a discrepancy between practitioners feeling they were supporting patients (subtheme A3) while recognizing that the CID-PPLM could also be restrictive or even invasive (theme C). These inconsistencies highlight the complexity of contemporary clinical practice, the 
idiosyncratic needs and behaviors of patients, and the importance of the practitioner's intuition to make care related decisions.

Implications for clinical practice include overt practitioner awareness of themes and subthemes. Conscious awareness of these factors - before implementing the protocol may lead to increased rapport and therapeutic effectiveness. Understanding therapeutic mechanisms concerning how and why the protocol might be effective can enable the practitioner to use these factors to benefit patients. Moreover, emerging themes and subthemes can be used to create derivations and modify the CID-PPLM to provide clinicians with additional flexibility to best serve music therapy service users. Additionally, themes and subthemes can be used as frameworks to create new PPLM-focused music therapy protocols based from the results of this qualitative investigation.

\section{Limitations}

Limitations of the study include a narrow scope of clinical work. All practitioners who participated in interviews worked with solid organ transplant patients or cancer patients who were typically hospitalized for only a few days, which is common in the healthcare system of the United States (Boyle, 2008; Miller, 2008). Other music therapists working in more long-term inpatient or outpatient medical settings - or practicing in different countries where longer inpatient hospitalizations are the norm - may have different perceptions and interpretations of the data. Another potential limitation is the author's previously established relationships with participants, who, despite the author's efforts, may have answered interview questions in a non-critical manner. However, participants did critique the protocol and offered ways to improve it, as evidenced in Theme C. Finally, as the CID-PPLM (Hogan \& Silverman, 2015) was developed specifically for music therapists who possess both music and therapeutic skills, practitioners must be qualified music therapists. Non-music therapy practitioners using the CID-PPLM (or PPLM) may have different results due to the unique academic and clinical training of music therapists.

\section{Recommendations for Future Research}

Suggestions for future research include more flexible research protocols, wherein practitioners can use their clinical intuition to alter the intervention based on idiosyncratic patient responses and behaviors. More flexible protocols might be especially useful for practitioners who have more clinical experience, as they will likely possess additional intuition based from their previous experiences. Due to the structure and applicability of the CID-PPLM for less experienced practitioners (subtheme B3), perhaps researchers could investigate using the CID-PPLM as a training and developmental tool to advance therapeutic interaction skills. Additionally, interviewing patients who received CID-PPLM could provide valuable data concerning their perceptions of the protocol. As patients' values and preferences constitute a component of EBP, a qualitative study with patients would be valuable to more holistically understand the CID-PPLM. Finally, with increasing interests in health musicking and music medicine, studies are needed to further differentiate music therapy and other modalities using music.

\section{Conclusion}

The purpose of this qualitative study was to understand practitioners' perspectives of the CID-PPLM protocol. Three themes and eight supporting sub-themes emerged and indicated that practitioners had positive, as well as constructive, perceptions of the CID-PPLM. Results of the study may provide a framework for the development of innovative protocols to meet the clinical needs of music therapy service users. Additional investigations using all research paradigms and data types are warranted. 


\section{References}

Boyle, M. E. (2008). Capturing unique and important data within the brief medical music therapy service model. Paper presented at the 34th Conference of the Canadian Association for Music Therapy, Quebec City, Canada.

Braun, V., \& Clarke, V. (2006). Using thematic analysis in psychology. Qualitative Research in Psychology, 3, 77-101, https://dx.doi.org/10.1191/1478088706qp063oa.

Centre for Evidence-Based Medicine. (2017). Levels of evidence. Retrieved from http://www.cebm.net/oxford-centre-evidence-based-medicine-levels-evidence-march-2009/

Craske, M. G. (2010). Cognitive-behavioral therapy. Washington, DC: American Psychological Association.

Edwards, J. E. (2012). We need to talk about epistemology: Orientations, meaning, and interpretation within music therapy research. Journal of Music Therapy, 49, 372-394, https://dx.doi.org/10.1093/jmt/49.4.372.

Fredenburg, H. A., \& Silverman, M. J. (2014). Effects of cognitive-behavioral music therapy on fatigue with patients on a blood and marrow transplantation unit: A mixed methods pilot study. The Arts in Psychotherapy, 41, 433-444, https://dx.doi.org/10.1016/ j.aip.2014.09.002.

Haack, B. W., \& Silverman, M. J. (2016). Effects of guitar accompaniment style within patient preferred live music on mood and pain with hospitalized patients on a solid organ transplant unit: A three group randomized pilot study. The Arts in Psychotherapy, 52, 32-40, https://dx.doi.org/10.1016/j.aip.2016.09.005.

Hogan, T., \& Silverman, M. J. (2015). Coping-infused dialogue through patient-preferred live music: A medical music therapy protocol and randomized pilot study. Journal of Music Therapy, 52, 420-436, https://dx.doi.org/10.1093/jmt/thv008.

Lazarus, R. S. (2006). Stress and emotion: A new synthesis. New York: Springer.

Lazarus, R., \& Folkman, S. (1984). Stress, appraisal, and coping. New York, NY: Springer.

Letwin, L., \& Silverman, M. J. (2017). No between-group difference but tendencies for patient support: A pilot study of a resilience-focused music therapy protocol for adults on a medical oncology/hematology unit. The Arts in Psychotherapy, 55, 116-125, https://dx.doi.org/ 10.1016/j.aip.2017.06.002.

Mansell, W., Carey, T. A., \& Tai, S. J. (2013). A transdiagnostic approach to CBT using method of levels therapy: Distinctive features. York, NY: Routledge.

Miller, K. (2008). Treatment planning and documentation in single-session and high-turnover situations. Paper presented at the $12^{\text {th }}$ World Congress of Music Therapy, Buenos Aires, Argentina.

Robb, S. L. (2003). Coping and chronic illness: Music therapy for children and adolescents with cancer. In S. L. Robb (Ed.), Music therapy in pediatric healthcare: Research and evidencebased practice (pp. 101-136). Silver Spring, MD: American Music Therapy Association.

Rosenow, S., \& Silverman, M. J. (2014). Effects of single session music therapy on hospitalized patients recovering from a bone marrow transplant: Two studies. The Arts in Psychotherapy, 41, 65-70, https://dx.doi.org/10.1016/j.aip.2013.11.003.

Rubin, A. (2008). Practitioner's guide to using research for evidence-based practice. Hoboken, NJ: John Wiley \& Sons.

Sackett, D. L., Straus, S. E., Richardson, W. S., Rosenberg, W., \& Haynes, R. B. (2000). Evidence-based medicine: How to practice and teach EBM. London, England: Churchill Livingston.

Semmer, N. K., \& Meier, L. L. (2009). Individual differences, work stress and health. In C. L. Cooper \& M. J. Schabracq (Eds.), International handbook of work and health psychology ( (pp. 99-121). https://dx.doi.org/10.1002/9780470682357.ch6. 
Silverman, M. J., Letwin, L., \& Nuehring, L. (2016). Patient preferred live music with adult medical patients: A systematic review to determine implications for clinical practice and future research. The Arts in Psychotherapy, 49, 1-7, https://dx.doi.org/10.1016/ j.aip.2016.05.004.

Silverman, M. J., Nuehring, L., \& Letwin, L. (2016). Effects of coping-infused dialogue through patient preferred live music on affective state and pain with adult medical inpatients: Two randomized effectiveness studies. International Journal of Music and Performing Arts, 4, 19-30, https://dx.doi.org/10.15640/ijmpa.v4n2a2.

Stige, B., Malterud, K., \& Midtgarden, T. (2009). Toward an agenda for evaluation of qualitative research. Qualitative Health Research, 19, 1504-1516, https://dx.doi.org/ 10.1177/1049732309348501.

Yates, G. J., \& Silverman, M. J. (2015). Immediate effects of single-session music therapy on affective state in patients on a post-surgical oncology unit: A randomized effectiveness study. The Arts in Psychotherapy, 44, 57-61, https://dx.doi.org/10.1016/j.aip.2014.11.002. 